\title{
Detection and study of Dark Electric Matter Objects (DAEMONs)
}

\section{E.M.Drobyshevski*}

Ioffe Physico-Technical Institute, Russian Academy of Sciences, 194021 St-Petersburg, Russia

E-mail: emdrob@mail.ioffe.ru

Abstract: We proceeded from the hypothesis of the CDM particles being the relic Planckian elementary black holes. Considerations of a very general nature permit an assumption that they should carry an electric charge $\sim 10 \mathrm{e}$. Such negatively charged DArk Electric Matter Objects, daemons, are nuclear-active particles, because when capturing light atomic nuclei, they mediate their fusion, whereas the energy of capture of heavy nuclei is enough for their partial disintegration. Planckian fields are strongest in Nature, so that a daemon-containing proton has to decay in a short time. 3-year-long observations performed with a detector consisting of two separated $\mathrm{ZnS}(\mathrm{Ag})$ scintillating screens and making use of ideas on the daemon-catalyzed proton decay and heavy nucleus disintegration have revealed several slowly moving $(\sim 30-15-5 \mathrm{~km} / \mathrm{s})$ dynamical daemon populations captured by the Solar system and evolving from one to another. The particles are captured presumably from the Galactic disc CDM background as they slow down in passing through the Sun and their orbits are perturbed by planets. Their flux precipitating onto the Earth mainly from the near-Earth almost circular heliocentric orbits is $\geq 10^{-9} \mathrm{~cm}^{-2} \mathrm{~s}^{-1}$ at such a velocity as $\sim 10-15 \mathrm{~km} / \mathrm{s}$. The flux undergoes strong variations with a half-year period due to the Earth's passage through the shadow and "anti-shadow" created by the moving Sun in the Galactic daemon background. A minor part of the daemons build up in the Sun and the Earth, which, in view of fast decay of a daemon-containing proton (measured by us to be $\sim 1 \mu \mathrm{sec}$ ), could account for many phenomena that have long defied interpretation.

KEYwords: Dark Matter detection, DM in Solar System, Planckian objects, proton decay.

\footnotetext{
${ }^{*}$ Speaker.
} 


\section{Introduction}

The issue of the Dark Mass (DM) was first recognized about 70 years ago and still remains an unsolved enigma. Assuming our Universe to have started from Planckian scales, it appears only natural to conjecture that the larger part of its mass still resides in Planckian objects [1]. These are elementary black holes with $M \sim 3 \times 10^{-5} \mathrm{~g}$ and $r_{g} \sim 10^{-33} \mathrm{~cm}$; indeed, a smaller mass could not have been confined within the gravitational radius $r_{g}$, because the Compton wavelength of this mass is in excess of $r_{g}$. Some authors believe that such objects are stable and may represent, in particular, the final product of evaporation of more massive black holes [1, 2, 3]. Based on considerations of a very general nature, one can also assume that these objects carry an electric charge; their gravitation is capable of counteracting the repulsive action of a charge of up to $Z \mathrm{e} \approx 10 \mathrm{e}$ 团. The first to consider the properties of Planckian black holes were apparently Markov (quantum maximons) [4 and Stanyukovich (Planckeons) [0] (half a dozen names have been proposed since, such as Newtorites, pyrgons, etc. [6]). Both authors allowed the possibility of conversion of these particles to ordinary matter. In view of the huge penetrating ability of such massive particles (for $V \sim 10^{6}-10^{7} \mathrm{~cm} / \mathrm{s}$, they could pass a distance $\sim 10^{7} \mathrm{~km}$ in a solid body), Markov was skeptical about the possibility of their detection.

\section{Daemons and Their Properties}

We called the Planckian objects daemons (which stands for DArk Electric Matter Objects) and suggested that negative daemons are nuclear-active particles, which makes them detectable. Indeed, the ground state of such massive particles with $Z=10$ lies within any nucleus starting with ${ }^{2} \mathrm{D}$, and starting with $Z_{n}>24 / Z$ even inside a proton in the nucleus [7]. Also, for such nuclei as Fe or $\mathrm{Zn}$ the binding energy $W \approx 1.8 Z Z_{n} A^{-1 / 3} \mathrm{MeV}$ becomes as high as $120-130 \mathrm{MeV}$. Release of this energy in the daemon capture by a nucleus results in a boiling up of the latter and ejection from it of about 10-15 nucleons, as well as of their clusters (before this, atomic shell and refilling electrons are ejected by the Auger mechanism and internal conversion). All these particles, carrying an energy $\geq 10 \mathrm{MeV}$, are capable of exciting a very strong scintillation. Moreover, on entering protons inside the remainder of the nucleus de-excited by evaporation of particles, daemon catalyzes their decay, one after another, in a comparatively short time $\left(\sim 10^{-7}-10^{-6} \mathrm{~s}\right)[0,8]$ (it is appropriate to recall here the catalysis of proton decay by a magnetic monopole discussed by Rubakov [9; the fields generated by the daemon are, by definition, the strongest in Nature; therefore, although the proton decay mechanism cannot be specified presently in detail, there are grounds to believe that the proton decay stimulated by a daemon should be more efficient than that by a monopole).

After the proton decays have lowered $Z_{n}$ of the remainder of the captured nucleus down to $Z_{n}<Z$, the daemon is capable of capturing another nucleus, followed again by emission of new particles, and so on.

For a DM density in the galactic halo $\sim 0.3 \mathrm{GeV} / \mathrm{cm}^{3}$, the flux of daemons with a velocity $\sim 250 \mathrm{~km} / \mathrm{s}$ at the Earth should be only $f_{\infty} \approx 4 \times 10^{-13} \mathrm{~cm}^{-2} \mathrm{~s}^{-1}$, i.e., one 
particle per year per $\sim 10 \mathrm{~m}^{2}$. Bahcall et al [10] estimate the velocity dispersion of the galactic disk DM particles as $\sim 4-30 \mathrm{~km} / \mathrm{s}$. In traversing the Sun, daemons moving with such a velocity are slowed down efficiently and captured by it [11]. The capture takes place originally into strongly elongated orbits with perihelia inside the Sun [12]. The aphelia of these orbits (as of the SEECHOs, see below) are oriented predominantly in the direction of the shadow and antishadow, which appear because of the Suns motion relative to the background daemon population in the galactic disk (the antishadow is created as a result of the particles which were slowed down by the Sun falling back on the Sun and traversing it again).

In subsequent daemon passages through the Sun, the orbits contract gradually, with the daemons becoming confined within the Sun and accumulating there to form a daemon kernel. The daemon-stimulated proton decay could account partially for the solar energetics, the deficiency of the electronic neutrinos, and appearance of neutrinos of nonelectronic flavor (assuming certainly that only negative daemons build up in the Sun) [13.

If a daemon moving in an orbit with an intrasolar perihelion passes through the sphere of action of the Earth (or of any other planet), there is a high probability for the perihelion to move out of the Sun. As a result, daemons will build up in stable, strongly elongated, Earth-crossing heliocentric orbits (SEECHOs). Straightforward estimates show that the flux of daemons from these orbits onto the Earth may exceed $f_{\infty}$ by $3-5$ orders of magnitude [12]. In subsequent crossings of the Earths sphere of action, daemons transfer from the SEECHOs to near-Earth, almost-circular, heliocentric orbits (NEACHOs). The points at which NEACHOs cross the Earths orbit should lie naturally in the "shadow" and "antishadow" regions.

\section{Ideology of the Daemon Detection. Our Detector}

The above reasoning forms a basis of the ideology of our experiment on detection of at least some indications of the existence of daemons [14]:

1.One should focus on a search for low-velocity $(\leq 30 \mathrm{~km} / \mathrm{s})$ objects which are members of the Solar system (their flux is relatively high).

2. One should employ the scintillation technique, and the scintillators should be preferably substances with a comparatively large atomic number and an as low as possible nuclear excitation level, so as to facilitate its capture by a negative daemon. Recall the first excitation levels of some nuclei: ${ }^{12} \mathrm{C}-4.4 \mathrm{MeV},{ }^{14} \mathrm{~N}-2.3 \mathrm{MeV},{ }^{16} \mathrm{O}-6 \mathrm{MeV},{ }^{56} \mathrm{Fe}$ - $0.85 \mathrm{MeV},{ }^{64} \mathrm{Zn}-1 \mathrm{MeV},{ }^{133} \mathrm{Cs}-81 \mathrm{keV}$; considered from this standpoint, the classical scintillator $\mathrm{ZnS}(\mathrm{Ag})$, which possesses also the highest light yield of up to $28 \%$ of absorbed energy, appears the most preferable of all. As follows from straightforward estimates taking into account the displacement of the scintillator nuclei toward a slowly moving negative daemon with $Z=10$, the path of the latter in $\mathrm{ZnS}(\mathrm{Ag})$ at a velocity of $15 \mathrm{~km} / \mathrm{s}$ until capture of a $\mathrm{Zn}$ nucleus is only $\sim 0.5 \mu \mathrm{m}$ (for $Z_{\text {eff }}=1$, the mean path increases to $\sim 5$ $\mu \mathrm{m})$. 
The detector designed by us has four identical modules [14]. Each module consists of two transparent, mutually light-isolated polystyrene plates, $4 \mathrm{~mm}$ thick and $0.5 \times 0.5 \mathrm{~m}^{2}$ in area, arranged horizontally at a distance of $7 \mathrm{~cm}$ from one another. They are mounted at the center of a cubic, tinned-iron $(0.3-\mathrm{mm}$ thick Fe coated on both sides with $2 \mu \mathrm{m}$ $\mathrm{Sn}$ ) case $51 \mathrm{~cm}$ on a side, whose top side is covered by black paper. Each plate is viewed on its side by a FEU-167 PM tube ( $\oslash 100$ dia. photocathode) from a distance of $22 \mathrm{~cm}$. The plates are coated on the underside with $\sim 3.5 \mathrm{mg} / \mathrm{cm}^{2}$ layer of $\mathrm{ZnS}(\mathrm{Ag})$ powder. We purposefully made our detector asymmetric to stress the contrast between the signatures of up- and downward moving objects.

The signals from the top and bottom PM tubes were fed into a double-trace digital oscilloscope and observed within the intervals of $\pm 100 \mu$ s from the trigger pulse from the top PM tube. If the bottom trace also had a signal, this event was recorded in the computer memory. Only those events were processed which had only one signal on each trace, and whose top signal began with a time shift $|\Delta t|>0.5 \mu$ s relative to the bottom one, thus excluding simultaneous signals due to cosmic rays (they were used to compare the sensitivities of the top and bottom channels). It was found that the shape of the $\mathrm{ZnS}(\mathrm{Ag})$ signals depends strongly on the kind of the radiation exciting the scintillator. For instance, the signals produced by $\alpha$-particles of ${ }^{238} \mathrm{Pu}$ pass through a broad maximum at about 2.5 $\mu \mathrm{s}$ from the beginning; we called them Heavy Particle Scintillations (HPSs). Cosmic ray signals do not differ in shape from the intrinsic PM tube noise, whence the name Noise-Like Scintillations (NLSs).

\section{Experimental Results}

Our approach consisted in looking for events in the top and bottom $\mathrm{ZnS}(\mathrm{Ag})$ screens which would be correlated in $\Delta t$. It appears reasonable that the distribution of paired events, $N(\Delta t)$, should not differ from a constant level if it reflects independent, pure background events in both screens. Only the daemon component should make it different from $N(\Delta t)$ $=$ const.

\section{4a. Discovery of Daemons and Estimation of Their Flux}

Figure 1 displays a $N(\Delta t)$ distribution for 417 events recorded in March 2000 [14] (these data were obtained with the signal fed from the PM tube anode to the oscilloscope through an inductance of $4.69 \mathrm{mH}$, which favored preferential selection of HPSs against the NLSs). Application of the $\chi^{2}$ criterion shows that the distribution $N(\Delta t) \neq$ const at a confidence level (C.L.) of $1-\alpha \approx 99 \%$. If we take only the HPSs triggered by an upper trace signal, the number of events decreases to 231 , but the confidence level of $N(\Delta t) \neq$ const increases to $1-\alpha \approx 99.7 \%$. In both cases, a maximum in the $20<\Delta t<40 \mu \mathrm{s}$ bin is present. In the first case, it contains 62 events, which exceeds the mean level by $2.58 \sigma$, and in the second, it still retains 41 events, but it is in excess of the mean level by $2.84 \sigma$, i.e., its significance is $99.5 \%$. 


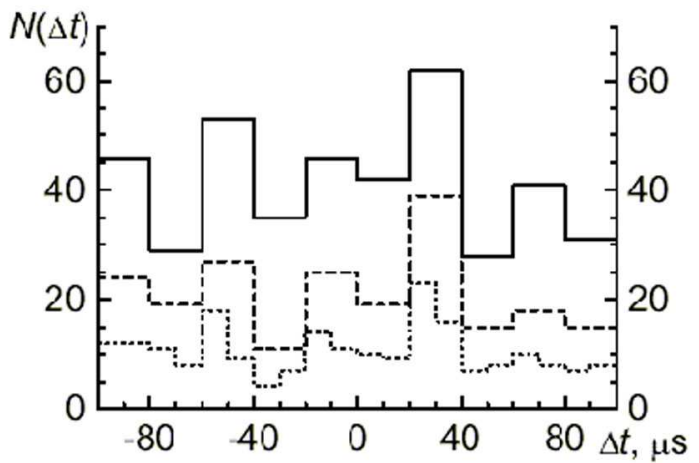

Figure 1: (-) Distribution $N(\Delta t)$ of pair scintillation events on their time shift (relative to the upper channel events). (_--) Similar distribution which takes into account only HPS (heavy-particle scintillation) type events at the upper channel. (...) The $10 \mu \mathrm{s}$ bin HPS distribution.

Assuming this maximum to be due to a downward-propagating flux, its value for a detector area of $1 \mathrm{~m}^{2}$ amounts to $f_{\oplus} \approx$ $0.7 \times 10^{-9} \mathrm{~cm}^{-2} \mathrm{~s}^{-1}$. Including the particle flux beyond this maximum and from below $(\Delta t<0)$, as well as the reduced lightcollection efficiency from the periphery of the screens, may increase $f_{\oplus}$ by nearly an order of magnitude.

\section{4b. Discrimination of the Upward Flux}

The upward-propagating flux can be discriminated in the given detector design in an as obvious way by turning the modules upside down. However, stimulated by the desire to obtain statistically significant, uniform results in as short a time as possible, we, rather than performing such an experiment, chose another approach 10. The fact is that the width of an HPS scintillation should depend somehow for a number of fairly clear reasons on whether the daemon carrying the nucleus captured in $\mathrm{ZnS}(\mathrm{Ag})$ moves, say, downward (i.e., into free space) or upward (into the polystyrene). In the latter case, if the daemon velocity is high enough, part of the nucleons from the excited nucleus will come to rest in the polystyrene without reaching the $\mathrm{ZnS}(\mathrm{Ag})$ layer, with the result that the HPS may become narrower. In view of the $\mathrm{ZnS}(\mathrm{Ag})$ powder being opaque to its intrinsic radiation, the HPS width should actually depend nonmonotonically not only on the daemon velocity but on the $\mathrm{ZnS}(\mathrm{Ag})$ layer thickness too, etc.

Figure 2 [15] displays a standard $N(\Delta t)$ distribution (Fig. 2c), as well as the distributions $N_{w}(\Delta t)$ for broad events (the scintillation area in an oscillogram normalized against the amplitude is larger than the mean for the given set of events), Fig. 2a, and $N_{n}(\Delta t)$ for narrow events - Fig. 2b. To stress the effect, Fig. 2d presents also the sum $N_{w}(\Delta t)+$ $N_{n}(-\Delta t)$. If we approximate the $N_{w}(\Delta t)$ and $N_{n}(\Delta t)$ distributions by straight lines, the latter will be found to have slopes of opposite signs.

Whence it follows that the HPS width does indeed depend on the sign of $\Delta t$, i.e., on the direction of daemon propagation, which provides not only evidence for the existence of a flux from below but additional arguments for the existence of daemons generally (because the observed differences between $N_{w}(\Delta t)$ and $N_{n}(\Delta t)$ follow from the daemon hypothesis).

\section{4c. Identification of Daemon Population in the Near-Earth Almost Circular Orbits. The Proton Decay Time. The Daemon Kernel of the Earth}

The velocity derived from the 7-cm spacing between the $\mathrm{ZnS}(\mathrm{Ag})$ screens and the time 20 $<\Delta t<40 \mu$ s turns out bafflingly low, $V \sim 3.5-1.8 \mathrm{~km} / \mathrm{s}$ (and not over $4-7 \mathrm{~km} / \mathrm{s}$, if one takes the possible trajectory inclination into account). This difficulty is removed [14] if 


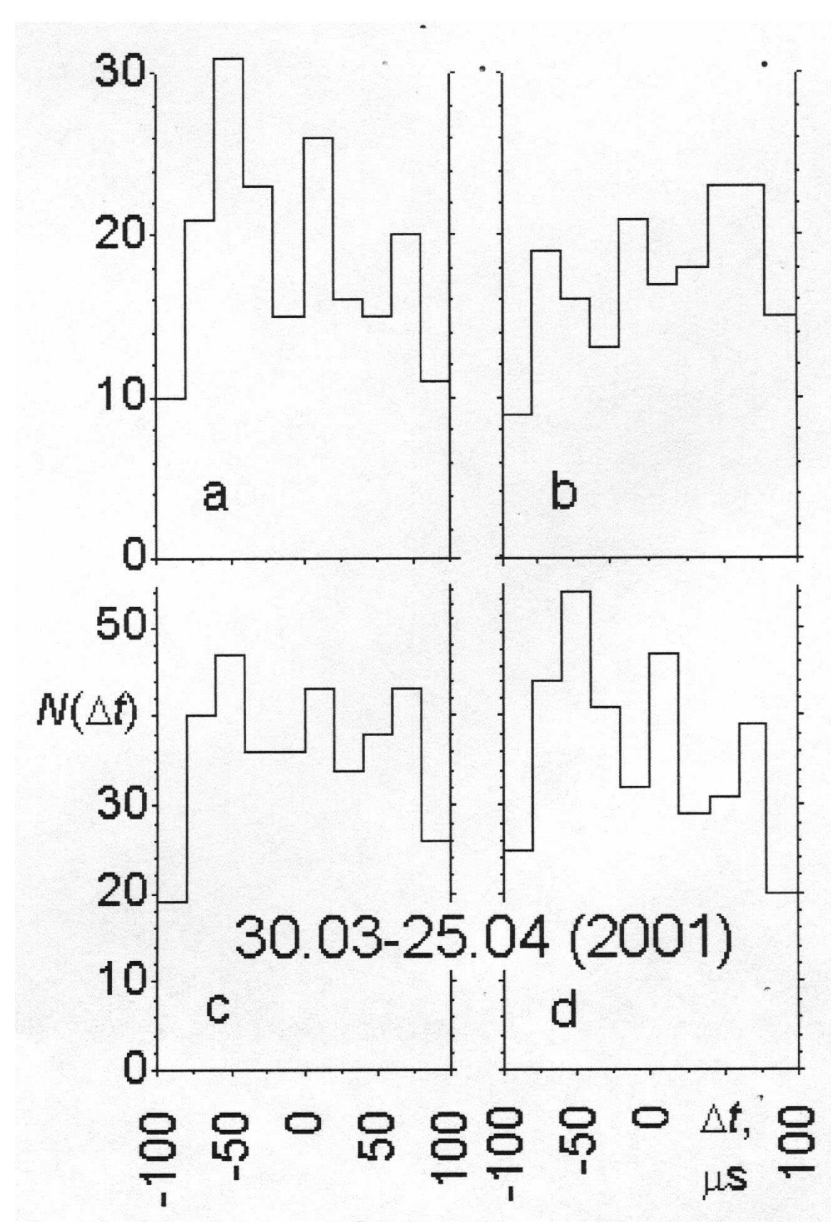

Figure 2: Statistics for shifted scintillations of upper and lower scintillators. Only HPS events at the upper scintillator are taken into account. (a) the wide HPS distribution $N_{w}(\Delta t)$; a maximum at $-80<\Delta t<-40 \mu$ s demonstrates an existence of the daemon flux going through the Earth upwards; (b) the narrow HPS distribution $N_{n}(\Delta t)$ (for the definition of $N_{w}(\Delta t)$ and $N_{n}(\Delta t)$ see Sec. $4 \mathrm{~b}$ ); (c) the combined distribution $N(\Delta t)=N_{w}+N_{n}$; (d) the inverted combined distribution $N_{w}(\Delta t)+$ $N_{n}(-\Delta t)$, which shows the dependence of the HPS width on the up/down direction of the daemon motion.

we allow that the downward-moving daemon which has captured a Zn nucleus in the top $\mathrm{ZnS}(\mathrm{Ag})$ layer cannot digest it down to $Z_{n}<Z$ by the time it crosses the bottom $\mathrm{ZnS}(\mathrm{Ag})$ layer, with the result that it traverses it without noticeable interaction. The digestion of $\sim 15$ protons in the remainder of the $\mathrm{Zn}$ nucleus becomes complete only by the time the daemon reaches the bottom lid of the tinned-iron case. Here the daemon captures a Fe or Sn nucleus with emission of electrons and protons. It is these electrons (and protons with $W \geq 3 \mathrm{MeV}$ ) that, on passing through $22 \mathrm{~cm}$ of air, reach the bottom scintillator and excite in it a scintillation. The distance $22+7 \mathrm{~cm}$ with $\Delta t=30 \mu$ s yields a velocity $\sim 10 \mathrm{~km} / \mathrm{s}$ (and up to $\sim 15 \mathrm{~km} / \mathrm{s}$, if the trajectory inclination is included), which fits well with the 
particles originating from the NEACHOs. A part of them, slowed down slightly in transit through the Earth, is captured into geocentric, Earth-surface-crossing orbits (GESCOs), which contract gradually to escape eventually under the Earths surface in 1-2 months. In April they produce maxima in $N(\Delta t)$ in the intervals $-80<\Delta t<-40 \mu$ s and $40<\Delta t<$ $80 \mu$ s (see Fig. 2) [15].

The above values of $\Delta t$ offer a possibility to estimate the daemon-stimulated proton decay time as $\sim 10^{-6} \mathrm{~s} 15$.

The daemons building up in the Earth form at its center a kernel $\sim 10^{19} \mathrm{~g}$ in mass and a few $\mathrm{cm}$ in size. The digestion of the iron nuclei diffusing into the kernel from outside is capable of providing the ${ }^{3} \mathrm{He}$ flux and one half of the heat flux (which is $\sim 20 \mathrm{TW}$ ) emerging from the Earth (the other $\sim 20 \mathrm{TW}$ are released in the disintegration of $\mathrm{U}$, $\mathrm{Th},{ }^{40} \mathrm{~K}$ ), which removes many of the problems associated with the structure of the inner iron core of the Earth, of its lower mantle, etc. that have piled up by now [16].

\section{Semiannual Variation of the Daemon Flux}

Interpretation of the fine features observed in $N(\Delta t)$ meets with difficulties associated with the relatively small statistics. Therefore, to reveal the character of the seasonal variations, we accepted an integral parameter $1-\alpha$, which is the statistical significance of the assumption that, by the $\chi^{2}$ criterion, the $N(\Delta t$ ) distribution (for $|\Delta t|<100 \mu \mathrm{s}$ ) cannot be approximated by $N=$ const. Figure 3 presents graphically the seasonal variation of 1 - $\alpha$ from April 2001 to March 2003 (see also [17]).

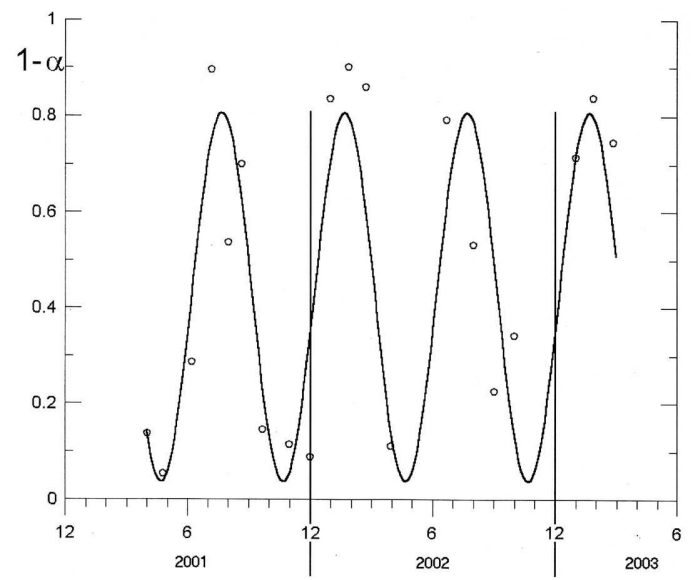

Figure 3: Semi-annual variation of $1-\alpha$, the (C.L.) to which the $N(\Delta t)$ distribution (at -100 $<\Delta t<100 \mu \mathrm{s}$ ) deviates from the constant level produced by background events.

with $1-\alpha$ varying during the half-year from 0.05 to 0.8
These data were processed for all the months by the same technique, which differed slightly from the ones employed when treating earlier results (Figs.1 and 2). For each of the four modules $\left(0.5 \times 0.5 \mathrm{~m}^{2}\right.$ in area), we chose monthly 90 HPS-type events with the maximum amplitude from the top screen. An additional condition was imposed in selecting events with paired top/bottom signals that the signal amplitude ratio from the bottom to the top screen exceeds 0.25 . A sine curve with a period $P=0.5$ yr was least-squares fitted to the experimental points in Fig. 3. The result is remarkable: the coefficient of correlation of the sine curve with the data points is 0.86 , and the statistical significance of this value is $99.9 \%$ (!), 


\section{Conclusions}

One can thus maintain that our experiments have detected indications of the existence of a flux of particles with properties which find readily explanation within the daemon approach, namely:

(1) The low velocities of objects, from $\sim 30$ to $\sim 10-15 \mathrm{~km} / \mathrm{s}$, with the latter figures corresponding to the fall of objects from NEACHOs, and $\sim 5-10 \mathrm{~km} / \mathrm{s}$, for objects escaping under the Earths surface.

(2) Enormous penetrating ability; the objects move both down- and upward, and the time taken up by their orbits to contract under the Earths surface is 1-2 months.

(3) A half-year flux periodicity indicating a preferred direction with respect to the daemon background in the galactic disk, which approximately coincides with the apex of the Suns motion relative to the nearest stars (the maxima in January-February-March and JulyAugust-September).

(4) The detection itself of the particles by our detector argues for the validity of the assumption of daemon-catalyzed proton decay. The time of this decay, estimated from an analysis of our experimental data, is $\sim 10^{-6} \mathrm{~s}$.

\section{Acknowledgments}

The author expresses deep gratitude to the Organizing Committee of the Conference and to the Russian Foundation for Basic Research (Grant 03-02-27288-z) for financial support.

\section{References}

[1] J.D.Barrow, E.J.Copeland, and A.R.Liddle, Phys. Rev. D 46 (1992) 645.

[2] A.D.Dolgov, P.D.Naselsky, and I.D.Novikov, astro-ph/0009407.

[3] S.Alexeyev, A.Barrau, G.Boudoul, O.Khovanskaya, M.Sazhin, Class. and Quant. Grav. 19 (2002) 4431 .

[4] M.A.Markov, Prog. Theor. Phys. Suppl. Extra Number (1965) 85; Zh. Eksp. Teor. Fiz. 51 (1966) 878.

[5] K.P.Stanyukovich, Doklady Acad. Scis. 168 (1966) 781 .

[6] H.V.Klapdor-Kleingrothaus, K.Zuber, Particle Astrophysics, IOP Publ. Ltd. (1997).

[7] E.M.Drobyshevski, Preprint PhTI-1663, St.Petersburg (1996), pp. 1-10.

[8] E.M.Drobyshevski, Mon. Not. Royal Astron. Soc. 311 (2000) L1.

[9] V.A.Rubakov, Pisma v ZhETF 33 (1981) 658; Nucl. Phys. B203 (1982) 311.

[10] J.H.Bahcall, C.Flynn, A.Gould, Astrophys. J. 389 (1992) 234.

[11] E.M.Drobyshevski, Mon. Not. Royal Astron. Soc. 282 (1996) 211.

[12] E.M.Drobyshevski, in: Dark Matter in Astro- and Particle Physics, H.V.Klapdor-Kleingrothaus, Y.Ramachers (eds.), World Scientific (1997), pp.417-424.

[13] E.M.Drobyshevski, Astron. E Astrophys. Trans. 23 (2004) in press; astro-ph/0205353. 
[14] E.M.Drobyshevski, Astron. E3 Astrophys. Trans. 21 (2002) 65.

[15] E.M.Drobyshevski, M.V.Beloborodyy, R.O.Kurakin, V.G.Latypov, K.A.Pelepelin, Astron. \& Astrophys. Trans. 22 (2003) 19 .

[16] E.M.Drobyshevski, Astron. ES Astrophys. Trans. 23 (2004), in press; estro-ph/0111042.

[17] E.M.Drobyshevski, M.E.Drobyshevski, T.Yu.Izmodenova, and D.S.Telnov, Astron. 8 Astrophys. Trans. 22 (2003) 263; astro-ph/0305597. 